\title{
LA ANTROPIZACIÓN DE LA COBERTURA VEGETAL EN LOS PAISAJES DEL ESTADO DE CHIAPAS, MÉXICO
}

\author{
Horacio Morales Iglesias ${ }^{1}$, Angel Guadalupe Priego Santander ${ }^{2}$, Manuel Bollo Manent ${ }^{3}$, \\ Mauricio José Ríos ${ }^{4}$ \\ Universidad de Ciencias y Artes de Chiapas y UNAM
}

\begin{abstract}
RESUMEN
El objetivo de la presente investigación es conocer y evaluar los niveles de modificación antrópica sobre la cobertura vegetal de los paisajes del estado de Chiapas, México. Para cumplir con este objetivo se aplicó un índice de presión al mapa de los paisajes físico-geográficos del estado de Chiapas, a escala 1: 250 000. Los resultados indican que la categoría baja ocupa el $29 \%$ de la superficie estatal; la clase alta 27\%; el nivel medio 26\%; la categoría muy baja $16 \%$; finalmente, el nivel muy alto abarca $2 \%$. La categoría alta se ubica en paisajes de planicie, piedemonte y valle, en clima cálido húmedo y cálido subhúmedo. La clase baja se localiza en montañas de origen tectónico-intrusivo, tectónico-acumulativo y tectónico-kárstico, en climas templado, semicálido húmedo a subhúmedo y cálido húmedo. El escenario de tendencia es preocupante, porque la categoría alta podría representar en el corto plazo 53\% de la superficie estatal.
\end{abstract}

Palabras clave: Paisajes; índice; antrópica; cobertura vegetal; Chiapas.

\section{THE ANTHROPIZATION OF THE VEGETATION COVER IN THE LANDSCAPES OF CHIAPAS STATE, MEXICO}

\begin{abstract}
The goal of this research is to understand and evaluate the levels of anthropic transformation on the vegetation cover in the landscapes of Chiapas, Mexico. To achieve this goal, a pressure index was applied to the map of the physical-geographical landscapes of the Chiapas State, in a 1:250 000 scale. The results indicate that low category occupies $29 \%$ of the surface area of the state; the high class $27 \%$, the medium level $26 \%$, the very low category $16 \%$; finally, the very high level covers $2 \%$. The high category is located in plains, piedmonts and valley landscapes in warm humid and subhumid climate. The low class is located in mountains of tectonic-intrusive, tectonic-acumulative and tectonic-karstic origin, in temperate, semiwarm humid to subhumid, and warm humid climates. The trend scenario is worrisome, because the high level could represent in the short term $53 \%$ of the surface area of the state.
\end{abstract}

Key words: Landscapes; index; anthropic; vegetation cover; Chiapas.

\footnotetext{
${ }^{1}$ Instituto de Investigación en Gestión de Riesgos y Cambio Climático, Universidad de Ciencias y Artes de Chiapas. E-mail: horacio.morales@unicach.mx

${ }^{2}$ Centro de Investigaciones en Geografía Ambiental, UNAM. E-mail: apriego@ ciga.unam.mx

3 Centro de Investigaciones en Geografía Ambiental, UNAM. E-mail: mbollo@ciga.unam.mx

4 Instituto de Investigación en Gestión de Riesgos y Cambio Climático, Universidad de Ciencias y Artes de Chiapas. E-mail: mauricio.jose@ unicach.mx
}

Fecha de recepción: 20 de septiembre de 2019. Fecha de aceptación: 29 de diciembre de 2019 


\section{INTRODUCCIÓN}

Ante la problemática ambiental que enfrenta la sociedad, y para lograr un desarrollo sustentable, es necesario realizar un uso racional del espacio; para ello, el ordenamiento ecológico representa un valioso aporte técnico-científico, debido a que orienta los esfuerzos sociales que regulan las actividades económicas y mantienen el patrimonio natural. Uno de los objetivos de este instrumento, es conocer los niveles de degradación que posee un determinado territorio; para ello, la geoecología representa una alternativa sólida, ya que evalúa este bajo una visión paisajista (PRIEGO SANTANDER et al., 2004; BOLLO MANENT y VELASCO MÉNDEZ, 2018).

La presente investigación considera al paisaje como como un espacio temporal complejo y abierto, que se origina y evoluciona en la interface naturaleza-sociedad, en un constante estado de intercambio de energía, materia e información, donde su estructura, funcionamiento, dinámica y evolución reflejan la interacción entre los componentes naturales (abióticos y bióticos), técnicoeconómicos y socio-culturales" (MATEO RODRÍGUEZ, 1984, 2008; BOLLO MANENT, 2018).

Como resultado de las transformaciones sociales y económicas que experimentan los territorios, los paisajes físico-geográficos manifiestan una pérdida de componentes, atributos y funciones, es decir, experimentan distintos niveles de degradación geoeocológica (MATEO RODRÍGUEZ y ORTIZ PÉREZ, 2001). Este fenómeno tiene efectos negativos, debido a que genera una pérdida de bio y geodiversidad, disminución de servicios ambientales, así como una mayor cantidad de territorios sujetos a procesos de riesgo por fenómenos geólogogeomorfológicos e hidro-meteorológicos. Ante este panorama, la comunidad científica considera conveniente elaborar evaluaciones rápidas y confiables, que permitan orientar las políticas ambientales de los distintos instrumentos de planificación territorial (FLORES DOMÍNGUEZ et al., 2017).

De acuerdo con PRIEGO SANTANDER et al., (2004), la vegetación es uno de los indicadores paisajistas que permite identificar de manera objetiva los grados de degradación que presenta el territorio; de esta manera, es posible identificar las entidades geográficas de mayor desarrollo económico, aquellas que albergan un mayor patrimonio natural, o que requieren de un proceso de restauración ecológica.

Como antecedentes, AGUIRRE et al., (2017) implementaron en la cuenca del río Dagua, Valle del Cauca, Colombia, una metodología para identificar unidades de paisaje antropogénicos, y la percepción que tienen los agentes sociales sobre estos. En México, BOLLO MANENT y VELASCO MÉNDEZ (2018) realizaron un diagnóstico ambiental bajo un enfoque geoecológico en el estado de Michoacán. Los resultados indican que Michoacán tiene una situación preocupante, ya que $19.1 \%$ del territorio se encuentra en estado crítico e inestable a crítico. Por su parte, FLORES DOMÍNGUEZ et al., (2017) evaluaron la degradación edáfica de los paisajes físico-geográficos del estado de Puebla; para ello, se aplicó el Índice de Degradación del Suelo (IDS). Los resultados señalan que menos del $20 \%$ del territorio poblano manifiesta suelos degradados.

Con base en lo anterior, el objetivo de la presente investigación es conocer los grados de transformación antrópica que tiene la cobertura vegetal de los paisajes físico-geográficos del estado de Chiapas. Los resultados obtenidos serán útiles en las distintas tareas de planificación territorial de la entidad.

\section{METODOLOGÍA}

\section{1 ÁREA DE ESTUDIO}

Chiapas se ubica en la parte más meridional de la República Mexicana, y junto con los estados de Guerrero, Veracruz, Oaxaca, Tabasco, Campeche, Yucatán y Quintana Roo, integra la Región Sur - Sureste de México (CONAPO, 1991). Al Norte colinda con el estado de Tabasco, al Sur con el Océano Pacífico, la parte Oeste con los estados de Oaxaca y Veracruz, al Este y Sureste con la República de Guatemala; sus coordenadas extremas son: $17^{\circ} 59^{\prime} 00^{\prime \prime}$ y $14^{\circ} 32^{\prime} 00^{\prime \prime}$ de latitud Norte, $90^{\circ} 22^{\prime} 00^{\prime}$ ' y $94^{\circ} 14^{\prime} 00^{\prime}$ ' de longitud Oeste. La figura 1 muestra la ubicación 
geográfica de Chiapas bajo un contexto continental y nacional.

FIGURA 1

Ubicación de la zona de estudio

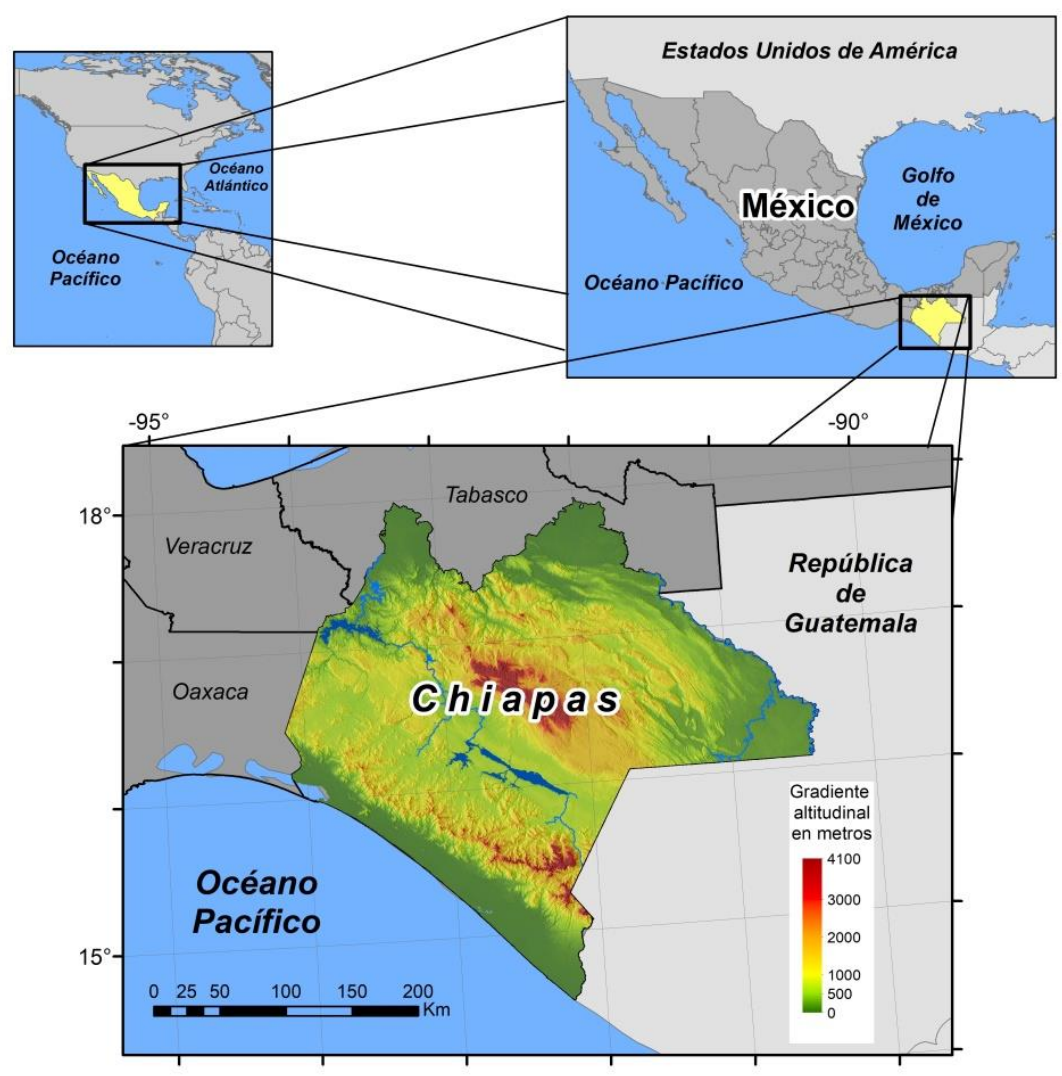

Fuente: Elaborado sobre información cartográfica de INEGI, 2018.

\subsection{MARCO TEÓRICO}

La presente investigación aborda el fenómeno de degradación ambiental desde una perspectiva geográfica; en este sentido, retoma los pensamientos teóricos expuestos por MATEO RODRÍGUEZ y ORTIZ PÉREZ (2001), quienes reconocen desde una aproximación físicogeográfica al paisaje o geosistema como la unidad de análisis espacial (ISACHENKO, 1973; MATEO RODRÍGUEZ, 1984, 2008; HASSE, 1986; FROLOVA, 2006; SHAW y OLFIELD, 2007; SEMENOV y SNYTKO, 2013; SEMENOV, 2014, BOLLO MANENT, 2018); en este sentido, consideran a la degradación geoecológica como "la pérdida de atributos y propiedades sistémicas que garantizan el cumplimiento de determinadas funciones, incluidas las socioeconómicas, y el potencial, los mecanismos de autorregulación y regeneración, así como de la capacidad productiva de los paisajes, por medio de la aparición y/o intensificación de los procesos geoecológicos" (MATEO RODRÍGUEZ y ORTIZ PÉREZ, 2001 p13). Bajo este punto de vista, PRIEGO SANTANDER et al., (2004) señalan que la vegetación es quizá el componente indicador o espejo del paisaje más significativo, ya que manifiesta de manera sintética los grados de degradación geoecológica presentes en un determinado espacio.

Por su parte, ROSETE VERGÉS et al., (2014) señalan que la vegetación es un regulador hidro-climático, tanto a nivel regional como local, forma parte de la riqueza biológica, dota de servicios ambientales a la sociedad humana; además, es un factor significativo en las emisiones de gases de efecto invernadero. Bajo estas consideraciones, la cobertura vegetal tiene un papel funcional preponderante, ya que regula los flujos de materia y energía en la estructura vertical y horizontal del paisaje; sin embargo, la perdida y degradación de este, genera procesos negativos 
que comprometen los servicios ecosistémicos (CUEVAS GARCÍA et al., 2010).

Por lo anterior, evaluar y conocer los grados de degradación paisajista a través de la antropización de la cobertura vegetal, permite zonificar de manera clara y objetiva el territorio para establecer políticas ambientales adecuadas; y con ello, contribuir a un desarrollo sustentable.

\subsection{MATERIALES Y MÉTODO}

Para evaluar los grados de transformación antropogénica que presenta la cobertura vegetal de los paisajes, se aplicó el Índice de Antropización de la Cobertura Vegetal (IACV) propuesto por SHISHENKO (1988). Este índice de presión (RODRÍGUEZ y LÓPEZ, 2008) ha sido aplicado en diversas regiones de Latinoamérica (PRIEGO SANTANDER et al., 2004; MATHEWS, 2011; AGUIRRE et al., 2017; CRUZ PAZ et al., 2018; BOLLO MANENT y VELASCO MÉNDEZ, 2018), bajo la siguiente ecuación:

$$
\mathrm{IACV}=\sum_{i=1}^{n} r i * A_{\mathrm{ij}}
$$

Donde:

IACV=Índice de antropización de la cobertura vegetal del paisaje.

ri= Grado de transformación antropogénica de los paisajes del tipo i de utilización.

Aij= Área dedicada al tipo de utilización i en el geocomplejo j.

$\mathrm{Aj}=$ Área de la unidad de paisaje $\mathrm{j}$.

Para cumplir con el objetivo, se consideró el mapa de los paisajes físico-geográficos del estado de Chiapas a nivel de Paraje Complejo en escala 1: 250 000, elaborado por MORALES IGLESIAS et al., (2017), e información sobre uso de suelo y vegetación del INEGI (2013). Bajo el marco lógico establecido por SHISHENKO (1988), cada uso de suelo o tipo de vegetación contenidos en una unidad de paisaje considera un valor ponderado o peso relativo ( 0.01 a 0.99$)$, según los grados de transformación; en sentido, los valores más altos representan una mayor degradación geoecológica y viceversa. Para el presente estudio, las ponderaciones fueron adaptadas a las condiciones económicas de la entidad; por tanto, los usos de suelo: urbano, agrícola y pecuario, poseen los valores más elevados (Tabla 1).

El método estableció una serie de pasos; el primero de ellos consideró una serie de operaciones matemáticas, en donde, los valores de ponderación de cada tipo de vegetación o uso de suelo fueron multiplicados por la superficie ocupada; posteriormente, se realizó la sumatoria de los productos obtenidos; después, el resultado se dividió entre el área total que ocupa la unidad de paisaje; finalmente, con los resultados obtenidos se procedió a la elaboración de un cartograma; para ello, se utilizó el método de clases iguales bajo cinco categorías: Muy alto, Alto, Medio, Bajo, Muy Bajo (PRIEGO SANTANDER et al., 2004). La figura 2 muestra los procedimientos realizados en un sistema de información geográfica (SIG), en este caso se aplicaron diversos módulos de ArcGIS Desktop (ESRI 2012). La discusión sobre la distribución de los grados de antropización consideró la propuesta de regionalización físico-geográfica de México elaborada por BOLLO MANENT et al., (2015). 
TABLA 1

Ponderación según tipo de vegetación y uso de suelo

\begin{tabular}{|c|c|c|c|}
\hline \multicolumn{3}{|c|}{ Tipos de vegetación según INEGI Serie V } & Ponderación bajo el \\
\hline \multirow{3}{*}{$\begin{array}{c}\text { Vegetación } \\
\text { primaria }\end{array}$} & 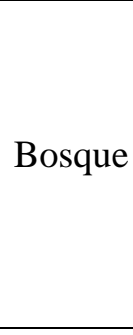 & $\begin{array}{l}\text { - } \text { Cedro (BB) } \\
\text { - Encino (BQ) } \\
\text { - Encino-Pino (BQP) } \\
\text { - Oyamel (BA) } \\
\text { - Pino (BP) } \\
\text { - Pino-Encino (BPQ) } \\
\text { - Mesófilo de Montaña (BM) }\end{array}$ & \multirow{3}{*}{0.01} \\
\hline & 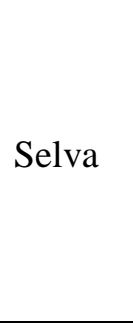 & $\begin{array}{ll}\text { - } & \text { Alta perennifolia (SAP) } \\
\text { - } & \text { Mediana subperennifolia } \\
& \text { (SMQ) } \\
\text { - } & \text { Baja caducifolia (SBC) } \\
\text { - } & \text { Baja espinosa subperennifolia } \\
& \text { (SBQ) } \\
\text { - } & \text { Baja perennifolia (SBP) } \\
\end{array}$ & \\
\hline & $\begin{array}{l}\text { Mangla } \\
\text { Popal ( } \\
\text { Pradera } \\
\text { Sabana } \\
\text { Sabanoi } \\
\text { Tular ( } \\
\text { Vegetac } \\
\text { Vegetac }\end{array}$ & $\begin{array}{l}\text { a }(\mathrm{VW}) \\
\text { osteras (VU) } \\
\text { (VG) }\end{array}$ & \\
\hline \multirow{3}{*}{$\begin{array}{c}\text { Vegetación } \\
\text { secundaria } \\
\text { arbórea }\end{array}$} & Bosque & $\begin{array}{ll}\text { - } & \text { Encino (VSA/BQ) } \\
\text { - } & \text { Encino-Pino (VSA/BQP) } \\
\text { - } & \text { Pino (VSA/BP) } \\
\text { - } & \text { Pino-Encino (VSA/BPQ) } \\
\text { - } & \text { Mesófilo de Montaña } \\
& (\mathrm{VSA} / \mathrm{BM})\end{array}$ & \multirow{3}{*}{0.2} \\
\hline & Selva & $\begin{array}{ll}- & \text { Alta perennifolia (VSA/SAP) } \\
\text { - } & \text { Baja caducifolia (VSA/SBC) } \\
\text { - } & \text { Baja espinosa caducifolia } \\
& \text { (VSA/SBQ) } \\
\text { - } & \text { Mediana subcaducifolia } \\
& \text { (VSA/SMS) } \\
\text { - } & \text { Mediana subperennifolia } \\
& \text { (VSA/SMQ) }\end{array}$ & \\
\hline & Mangla & & \\
\hline \multirow[t]{2}{*}{$\begin{array}{l}\text { Vegetación } \\
\text { Secundaria } \\
\text { arbustiva }\end{array}$} & & $\begin{array}{ll}\text { - } & \text { Alta perennifolia (VSa/SAP) } \\
\text { - } & \text { Baja caducifolia (VSa/SBC) } \\
\text { - } & \text { Baja espinosa caducifolia } \\
& (\mathrm{VSa} / \mathrm{SBQ}) \\
\text { - } & \text { Mediana subcaducifolia } \\
& (\mathrm{VSa} / \mathrm{SMS}) \\
\text { - } & \mathrm{Mediana} \text { subperennifolia } \\
& (\mathrm{VSa} / \mathrm{SMQ}) \\
\end{array}$ & \multirow[t]{2}{*}{0.3} \\
\hline & Mangla & & \\
\hline
\end{tabular}




\begin{tabular}{|c|c|c|c|}
\hline \multirow{2}{*}{$\begin{array}{l}\text { Vegetación } \\
\text { secundaria } \\
\text { herbácea }\end{array}$} & Bosque & $\begin{array}{ll}\text { - } & \text { Encino (VSh/BQ) } \\
\text { - Pino-Encino (VSh/BPQ) } \\
\text { - Mesófilo de Montaña (VSh/BM) } \\
\end{array}$ & \multirow[t]{2}{*}{0.4} \\
\hline & \multicolumn{2}{|r|}{ - Baja caducifolia (VSh/SBC) } & \\
\hline \multirow{8}{*}{ Uso de suelo } & \multirow[b]{2}{*}{ Pastizales } & Inducido (PI) & 0.60 \\
\hline & & Cultivado (PC) & 0.70 \\
\hline & Bosque & Cultivado (BC) & 0.70 \\
\hline & Palmar & Inducido (VPI) & 0.60 \\
\hline & \multirow[b]{2}{*}{$\begin{array}{l}\text { Agricultur } \\
\mathrm{a}\end{array}$} & Temporal (AT) & 0.80 \\
\hline & & Riego (AR) & 0.85 \\
\hline & \multicolumn{2}{|c|}{ Asentamientos humanos (AH) } & 0.90 \\
\hline & \multicolumn{2}{|c|}{ Zona urbana (ZU) } & 0.99 \\
\hline
\end{tabular}

Fuente: Elaboración propia

FIGURA 2

Procedimientos en el SIG para la obtención del mapa de antropización de la cobertura vegetal de los paisajes

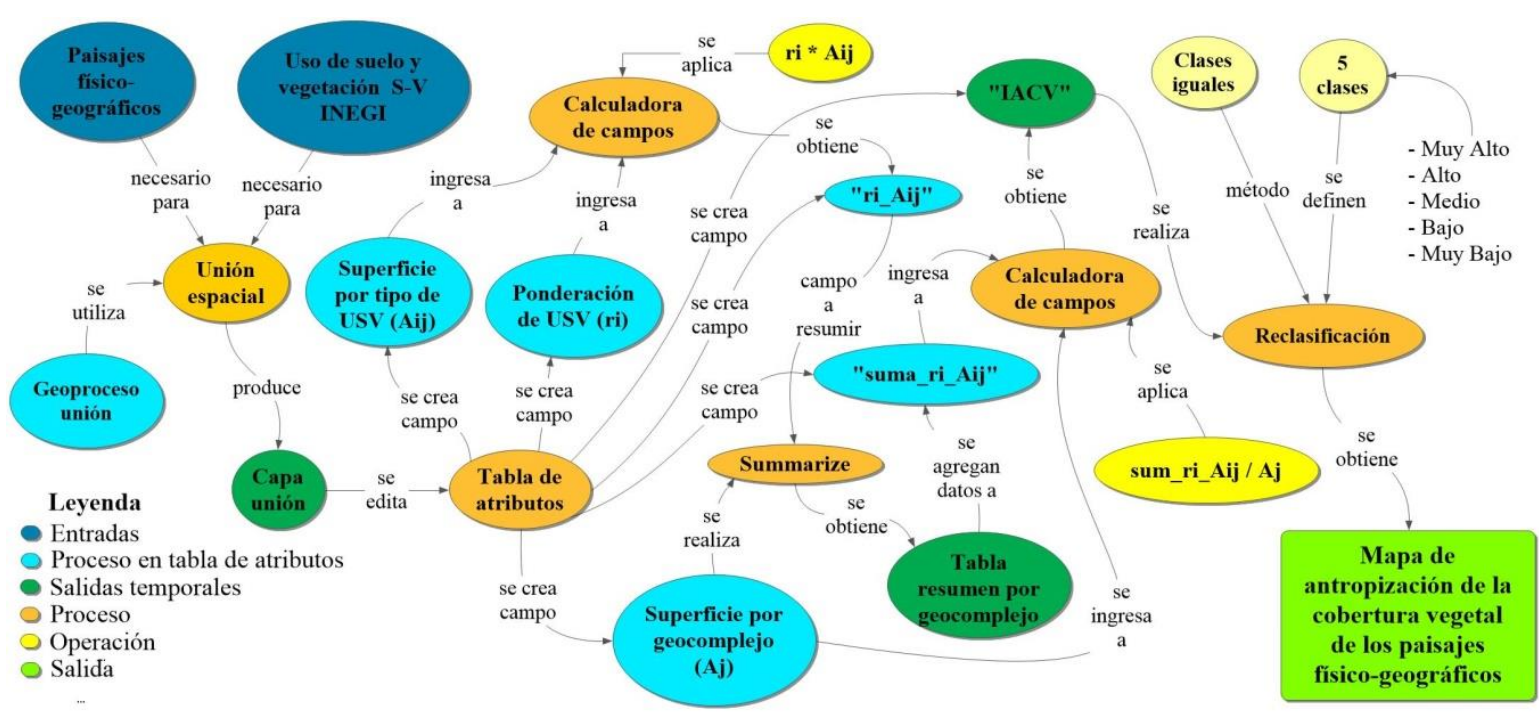

Fuente: Elaboración propia

\section{RESULTADOS Y DISCUSIÓN}

\subsection{COMPOSICIÓN DE LAS CATEGORÍAS DEL IACV}

De acuerdo con los tipos de vegetación y usos de suelo presentes en el territorio chiapaneco, se esclareció la composición de cada categoría del IACV. En los paisajes de categoría muy alta, el uso de suelo urbano y la agricultura de temporal ejercen un predominio territorial, ya que ambos usos abarcan 97\% del área total; le sigue el pastizal inducido con una superficie que representa $2 \%$; el último lugar es ocupado por la vegetación secundaria con apenas 1\% (Tabla 2). 
Por su parte, los pastizales cultivados y la agricultura de temporal ejercen una hegemonía espacial en los paisajes de categoría alta, debido a que ocupan $87 \%$ de la superficie; en segundo lugar se encuentra la agricultura de riego con 10\%; le sigue el uso de suelo urbano, la vegetación primaria y secundaria con apenas $3 \%$ (Tabla 2).

Respecto a los paisajes de categoría media, la vegetación secundaria arbustiva abarca $34 \%$ del área total; le sigue la agricultura de temporal con 26\%; la vegetación secundaria arbórea representa $20 \%$; mientras que el pastizal cultivado y la vegetación primaria engloban $9 \%$; por último, los pastizales inducidos ocupan $2 \%$ (Tabla 2).

Los paisajes de categoría baja tienen una composición más natural; en este sentido, la vegetación primaria ocupa 37\% del área total; le sigue la vegetación secundaria de tipo arbórea con 33\%; el tercer lugar lo representa la agricultura de temporal con 17\%; en una posición inmediata se encuentra la vegetación secundaria arbustiva con 8\%; finalmente, el pastizal inducido representa únicamente 5\% (Tabla 2).

Por su parte, la vegetación primaria ejerce un predominio territorial en los paisajes de categoría muy baja, al ocupar 58\% del total de la superficie; le sigue, la vegetación secundaria arbórea con $23 \%$; posteriormente, se ubica la vegetación secundaria de tipo arbustiva con $11 \%$; después, está presente el pastizal cultivado con tan sólo 7\%; finalmente, el pastizal inducido completa la composición con apenas $1 \%$ (Tabla 2).

Con base en lo anterior, los resultados revelan que la cobertura vegetal de la mayoría de los paisajes posee considerables grados de transformación antrópica; sin embargo, son pocos los geosistemas que poseen niveles extremos. La categoría baja ocupa poco más del $29 \%$ de la superficie estatal; el nivel alto $27 \%$; la categoría media $26 \%$; la clase muy baja $16 \%$; finalmente, el nivel muy alto abarca $2 \%$ (Tabla 2).

TABLA 2

Superficie ocupada por las categorías del IACV

\begin{tabular}{|c|c|c|}
\hline Categoría & Superficie en $\mathrm{km}^{2}$ & Porcentaje \\
\hline Muy baja & 11,478 & 16 \\
\hline Baja & 21,478 & 29 \\
\hline Media & 18,688 & 26 \\
\hline Alta & 19,452 & 27 \\
\hline Muy alta & 1,288 & 2 \\
\hline
\end{tabular}

Fuente: Elaboración propia

En cuanto al número de paisajes que ocupa cada categoría, destaca en primer lugar el nivel alto con 859 unidades, lo que representa poco más de $29 \%$ del total de paisajes que integran el territorio chiapaneco; le sigue la categoría baja con 761 , esto significa casi $26 \%$; después, se encuentra el rango medio con 703 unidades de paisaje, lo que equivale a más de 23\%; posteriormente, se ubica la categoría muy baja con 518 entidades, esto constituye $17 \%$ del territorio; el último lugar lo ocupa la categoría muy alta con 110 geosistemas, esto considera casi $4 \%$.

\subsection{LA ANTROPIZACIÓN DE LOS PAISAJES}

Según los resultados, y de acuerdo con el mapa de los paisajes físico-geográficos del estado de Chiapas, elaborado por MORALES IGLESIAS et al., (2017); la categoría alta se establece principalmente en paisajes de planicie, piedemonte y valle; estas unidades morfológicas consideran una génesis de tipo fluvial, fluvio-torrencial, fluvio-erosivo y tectónico-acumulativo, en climas cálido húmedo y cálido subhúmedo. Estas condiciones abióticas favorecen la presencia de un uso de suelo agrícola y pecuario (Tabla 3). La mayoría de estos paisajes se distribuyen en forma de amplios corredores por la Depresión Central de Chiapas, Montañas de Guatemala y 
Chiapas (Meseta Comiteca), Sierra Lacandona, Llanuras y Pantanos Tabasqueños, Llanura Costera de Chiapas, la parte más septentrional de las Montañas del Norte de Chiapas, así como en las estribaciones de la vertiente interna de las Sierras del Sur de Chiapas (Figura 3).

TABLA 3

Composición geomorfológica y vegetal de los paisajes predominantes de categoría alta

\begin{tabular}{|c|c|c|c|c|c|}
\hline \multirow{2}{*}{$\begin{array}{l}\text { Clave del paisaje según } \\
\text { MORALES IGLESIAS } \\
\text { et al., (2017) }\end{array}$} & \multirow{2}{*}{$\begin{array}{l}\text { Tipo } \\
\text { de } \\
\text { relieve }\end{array}$} & \multirow{2}{*}{$\begin{array}{l}\text { Rango de la } \\
\text { pendiente }\end{array}$} & \multirow{2}{*}{$\begin{array}{c}\text { Tipos de } \\
\text { vegetación o } \\
\text { usos de suelo }\end{array}$} & \multicolumn{2}{|c|}{ Área } \\
\hline & & & & $\mathrm{km}^{2}$ & $\%$ \\
\hline LXXXI.1 & PL: 3. & $\begin{array}{c}\text { Superficie plana }(< \\
\left.1^{\circ}\right)\end{array}$ & $\begin{array}{l}\text { (PC), (AT), } \\
\text { (AR). }\end{array}$ & $2,918.13$ & 28.91 \\
\hline LXVI.1 & PL: 2. & \multirow{3}{*}{$\begin{array}{c}\text { Suave a } \\
\text { medianamente } \\
\text { inclinado }\left(1^{\circ}-\right. \\
\left.10^{\circ}\right)\end{array}$} & $\begin{array}{l}(\mathrm{PC}),(\mathrm{Sa}) \\
\quad(\mathrm{AT})\end{array}$ & $1,604.89$ & 15.90 \\
\hline LXV.1 & PL: 2. & & $\begin{array}{l}\text { (PC), (AT), } \\
\text { (VSA/SAP). }\end{array}$ & 611.11 & 6.05 \\
\hline CVIII.1 & PL: 2. & & $\begin{array}{l}\text { (AT), (PC), } \\
\text { (AR.). }\end{array}$ & 602.16 & 5.97 \\
\hline XCVII. 2 & L: 2 . & \multirow{8}{*}{$\begin{array}{c}\text { Suave a } \\
\text { ligeramente } \\
\text { inclinado }\left(1^{\circ}-5^{\circ}\right) .\end{array}$} & $\begin{array}{l}\text { (AT), (VS } \\
\text { a/SBC). }\end{array}$ & 583.17 & 5.78 \\
\hline LXIV.2 & PM: 1. & & $\begin{array}{l}\text { (PC), (AT), } \\
\text { (PI). }\end{array}$ & 545.07 & 5.40 \\
\hline LXIII.2 & PM: 2. & & $\begin{array}{l}\text { (PC), (AT), } \\
\quad(\mathrm{AH}) .\end{array}$ & 538.03 & 5.33 \\
\hline CII. 2 & PM: 2. & & $\begin{array}{l}\text { (AT), (PC), } \\
\quad(\mathrm{AR}) .\end{array}$ & 526.74 & 5.22 \\
\hline CXVIII.2 & $\mathrm{V}: 3$. & & $\begin{array}{l}\text { (AT), (AR), } \\
\text { (PC). }\end{array}$ & 453.93 & 4.50 \\
\hline CI.2 & PM: 2. & & $(\mathrm{AT}),(\mathrm{PC})$ & 453.41 & 4.49 \\
\hline CXII.1 & PL: 3. & & $\begin{array}{l}\text { (PC), (AT), } \\
\quad(\mathrm{AR}) .\end{array}$ & 424.83 & 4.21 \\
\hline XCVIII.2 & L: 2. & & $\begin{array}{l}\text { (AT), (PI), } \\
\quad(\mathrm{PC}) .\end{array}$ & 420.03 & 4.16 \\
\hline CVI.1 & PL: 2. & $\begin{array}{c}\text { Suave a } \\
\text { medianamente } \\
\text { inclinado }\left(1^{\circ}-\right. \\
\left.10^{\circ}\right) .\end{array}$ & $(\mathrm{AT}),(\mathrm{PC})$ & 412.05 & 4.08 \\
\hline
\end{tabular}

Nota: L: Lomerío. PM: Piedemonte. PL: Planicie. V: Valle. 1: Complejo de colinas residuales, interfluvios y arroyos distributarios. 2: Complejo de colinas residuales, superficies y cauces. 3: Complejo de colinas residuales, vegas y cauces. Fuente: Elaboración propia. 
Por su parte, la categoría baja se ubica primordialmente en paisajes montañosos y de planicie. Los paisajes bajo la primer composición morfológica manifiestan una diversidad morfogenética, ya que consideran ambientes de tipo tectónico-intrusivo, tectónico-acumulativo y tectónicokárstico, en climas templado típico, semicálido húmedo a subhúmedo y cálido húmedo. Estas condiciones permiten la presencia de bosques templados y selvas húmedas; no obstante, la mayoría de estas comunidades vegetales considera un cierto grado transformación antrópica. Por su parte, los paisajes de ambientes deposicionales son más homogéneos, ya que solo responden a una génesis tectónico-acumulativa, bajo un clima cálido húmedo. Este escenario genera la presencia de selva húmeda (Tabla 4). Ambos tipos de paisajes se distribuyen principalmente en forma de corredores y parches por las Sierras del Sur de Chiapas, Sierras del Norte de Chiapas, Montañas de Chiapas y Guatemala (Altos de Chiapas), Sierra Lacandona y Peten Bajo (Figura 3).

TABLA 4

Composición geomorfológica y vegetal de los paisajes predominantes de categoría baja

\begin{tabular}{|c|c|c|c|c|c|}
\hline \multirow{2}{*}{$\begin{array}{l}\text { Clave del paisaje } \\
\text { según MORALES } \\
\text { IGLESIAS } \text { et al., } \\
\text { (2017) }\end{array}$} & \multirow{2}{*}{$\begin{array}{l}\text { Tipo de } \\
\text { relieve }\end{array}$} & \multirow{2}{*}{$\begin{array}{l}\text { Rango de la } \\
\text { pendiente }\end{array}$} & \multirow{2}{*}{$\begin{array}{c}\text { Tipos de } \\
\text { vegetación o } \\
\text { usos de suelo }\end{array}$} & \multicolumn{2}{|c|}{ Área } \\
\hline & & & & $\mathrm{km}^{2}$ & $\%$ \\
\hline XXVI.1 & & & $\begin{array}{l}\text { (VSA-a/BM), } \\
\text { (VSA/SAP), } \\
\text { (VSa/BPQ). }\end{array}$ & $4,324.45$ & 35.98 \\
\hline XXVIII.1 & & & $\begin{array}{c}(\mathrm{BM}) \\
(\mathrm{VSA} / \mathrm{SAP}) \\
(\mathrm{AT}),(\mathrm{BP})\end{array}$ & $1,855.43$ & 15.44 \\
\hline XIII.1 & M: 1. & fuertemente & $\begin{array}{c}\text { (AT), } \\
(\mathrm{VSa} / \mathrm{BPQ}), \\
\text { (BP). }\end{array}$ & $1,607.54$ & 13.37 \\
\hline XLIX.1 & & & $\begin{array}{l}\text { (VSA/SAP), } \\
\text { (SAP), (PC). }\end{array}$ & $1,295.38$ & 10.78 \\
\hline XXIV.1 & & & $\begin{array}{c}\text { (VSa-A/BPQ), } \\
(\mathrm{VSA} / \mathrm{BM}),(\mathrm{PI})\end{array}$ & $1,192.26$ & 9.92 \\
\hline LI.1 & & & (VSA-a/SAP). & $1,001.70$ & 8.33 \\
\hline LXX & PL:2 & $\begin{array}{c}\text { Suave a } \\
\text { medianamente } \\
\text { inclinado }\left(1^{\circ}-10^{\circ}\right) .\end{array}$ & $\begin{array}{c}(\mathrm{SAP}),(\mathrm{PC}) \\
(\mathrm{VSA}-\mathrm{a} / \mathrm{SAP})\end{array}$ & 742.56 & 6.18 \\
\hline
\end{tabular}

Nota: M: Montaña. PL: Planicie. 1: Complejo de cumbres, laderas y barrancos. 2: Complejo de colinas residuales, superficies y cauces. Fuente: Elaboración propia.

Respecto a la categoría media, esta se dispone en paisajes constituidos por un relieve de montaña y lomerío, cuya génesis responde a procesos tectónico-acumulativos, tectónico-carsificados y tectónico-intrusivos, en climas semicálido húmedo a subhúmedo, cálido húmedo y cálido subhúmedo. Sobre este escenario morfogenético se establecen bosques templados, selvas húmedas y secas; sin embargo, la mayoría de estas comunidades vegetales manifiestan considerables grados de modificación antrópica (Tabla 5). Los geosistemas bajo esta 
categoría se distribuyen por todo el estado en forma de parches; sin embargo, estos ejercen un predominio territorial en las Montañas del Norte de Chiapas y la Depresión Central de Chiapas (Figura 3).

TABLA 5

Composición geomorfológica y vegetal de los paisajes predominantes de categoría media

\begin{tabular}{|c|c|c|c|c|c|}
\hline \multirow{2}{*}{$\begin{array}{l}\text { Clave del paisaje } \\
\text { según MORALES } \\
\text { IGLESIAS et al., } \\
\text { (2017) }\end{array}$} & \multirow{2}{*}{$\begin{array}{l}\text { Tipo de } \\
\text { relieve }\end{array}$} & \multirow{2}{*}{$\begin{array}{l}\text { Rango de la } \\
\text { pendiente }\end{array}$} & \multirow{2}{*}{$\begin{array}{c}\text { Tipos de } \\
\text { vegetación o usos } \\
\text { de suelo }\end{array}$} & \multicolumn{2}{|c|}{ Área } \\
\hline & & & & $\mathrm{km}^{2}$ & $\%$ \\
\hline XXIV.1 & \multirow{6}{*}{ M: 1.} & \multirow{6}{*}{$\begin{array}{l}\text { Fuerte a muy } \\
\text { fuertemente } \\
\text { inclinado }(>\text { a } \\
\left.10^{\circ}\right) \text {. }\end{array}$} & $\begin{array}{c}\text { (PC), (AT), } \\
(\mathrm{VSa}-\mathrm{A} / \mathrm{BM}), \\
(\mathrm{VSa} / \mathrm{BPQ})\end{array}$ & $2,687.17$ & 27.53 \\
\hline XLVII.1 & & & $\begin{array}{c}\text { (PC), (VSA- } \\
\text { a/SAP). }\end{array}$ & $1,339.52$ & 13.73 \\
\hline XXV.1 & & & $\begin{array}{c}(\mathrm{AT}),(\mathrm{VSa} / \mathrm{BQ}), \\
(\mathrm{PI}) .\end{array}$ & $1,151.67$ & 11.80 \\
\hline XXVI.1 & & & $\begin{array}{l}\text { (PC), (AT), } \\
(\mathrm{VSa} / \mathrm{BPQ}), \\
(\mathrm{VSa} / \mathrm{SBC}) .\end{array}$ & $1,047.51$ & 10.73 \\
\hline XC.1 & & & $\begin{array}{c}(\mathrm{PI}),(\mathrm{AT}), \\
(\mathrm{VSa} / \mathrm{SBC}), \\
(\mathrm{VSA} / \mathrm{BPQ}) .\end{array}$ & 886.25 & 9.08 \\
\hline XLVIII.1 & & & $\begin{array}{c}(\mathrm{PC}) \\
(\mathrm{VSA} / \mathrm{SAP})\end{array}$ & 815.32 & 8.35 \\
\hline XCVII.2 & L: 2 . & $\begin{array}{c}\text { Suave a } \\
\text { ligeramente } \\
\text { inclinado }\left(1^{\circ}-5^{\circ}\right) .\end{array}$ & $\begin{array}{c}\text { (VSa-A/SBC), } \\
\text { (PC), (AT). }\end{array}$ & 659.70 & 6.76 \\
\hline L.1 & \multirow[b]{2}{*}{ M: 1.} & \multirow{2}{*}{$\begin{array}{l}\text { Fuerte a muy } \\
\text { fuertemente } \\
\text { inclinado }(>\text { a } \\
\left.10^{\circ}\right) .\end{array}$} & $\begin{array}{c}\text { (VSA-a/SAP), } \\
(\mathrm{PC}),(\mathrm{AT}) .\end{array}$ & 589.95 & 6.04 \\
\hline XIII.1 & & & $\begin{array}{c}(\mathrm{AT}), \\
(\mathrm{VSa} / \mathrm{BPQ}),(\mathrm{PI}) .\end{array}$ & 582.27 & 5.97 \\
\hline
\end{tabular}

Nota: M: Montaña. L: Lomerío. 1: Complejo de cumbres, laderas y barrancos. 2: Complejo de colinas residuales, superficies y cauces. Fuente: Elaboración propia.

La categoría muy baja se distribuye principalmente en paisajes montañosos de origen tectónico-intrusivo, tectónico-carsificado y tectónico-acumulativo; sin embargo, por la superficie de ocupación, es posible considerar a los geosistemas de planicie lacustre y palustre; sobre estas condiciones geomorfológicas, se establecen climas semicálido húmedo a subhúmedo y cálido 
húmedo. Esta situación morfo-climática favorece la presencia de bosque de niebla y selva alta perennifolia (Tabla 6). La mayoría de estos paisajes se distribuyen en forma de corredores por las Montañas del Sur de Chiapas, Sierra Lacandona, Llanura Costera de Chiapas, así como en pequeños parches por las Montañas del Norte de Chiapas (Figura 3).

TABLA 6

Composición geomorfológica y vegetal de los paisajes predominantes de categoría muy baja

\begin{tabular}{|c|c|c|c|c|c|}
\hline \multirow{2}{*}{$\begin{array}{l}\text { Clave del paisaje } \\
\text { según MORALES } \\
\text { IGLESIAS et al., } \\
\text { (2017) }\end{array}$} & \multirow{2}{*}{$\begin{array}{l}\text { Tipo de } \\
\text { relieve }\end{array}$} & \multirow{2}{*}{$\begin{array}{l}\text { Rango de la } \\
\text { pendiente }\end{array}$} & \multirow{2}{*}{$\begin{array}{c}\text { Tipos de } \\
\text { vegetación o } \\
\text { usos de suelo }\end{array}$} & \multicolumn{2}{|c|}{ Área } \\
\hline & & & & $\mathrm{km}^{2}$ & $\%$ \\
\hline XXVIII.1 & \multirow{4}{*}{ M: 1.} & \multirow{4}{*}{$\begin{array}{c}\text { Fuerte a muy } \\
\text { fuertemente } \\
\text { inclinado }\left(>\text { a } 10^{\circ}\right) .\end{array}$} & (BM), (BP). & $2,267.62$ & 36.97 \\
\hline XLIX.1 & & & (SAP). & $1,060.03$ & 17.28 \\
\hline LI.1 & & & (SAP). & $1,040.32$ & 16.96 \\
\hline XXVI.1 & & & $(\mathrm{SAP}),(\mathrm{BM})$ & 707.03 & 11.53 \\
\hline LXV.1 & PL: 2. & $\begin{array}{c}\text { Suave a } \\
\text { medianamente } \\
\text { inclinado }\left(1^{\circ}-10^{\circ}\right) .\end{array}$ & (SAP). & 534.86 & 8.72 \\
\hline LXXIX.1 & PL: 2. & $\begin{array}{l}\text { Superficie plana } \\
\qquad\left(<1^{\circ}\right)\end{array}$ & (Mg). & 523.28 & 8.53 \\
\hline
\end{tabular}

Nota: M: Montaña. PL: Planicie. 1: Complejo de cumbres, laderas y barrancos. 2: Complejo de colinas residuales, superficies y cauces. Fuente: Elaboración propia.

Finalmente, la categoría muy alta se ubica principalmente en paisajes de planicie y valle, cuya génesis responde a procesos de tipo fluvial, bajo un clima cálido subhúmedo. Estas condiciones favorecen la presencia de usos de suelo agrícola y pecuario (Tabla 7). Los paisajes con esta categoría se distribuyen primordialmente en forma de corredores y parches por la Depresión Central de Chiapas (Figura 3).

TABLA 7

Composición geomorfológica y vegetal de los paisajes predominantes de categoría muy alta

\begin{tabular}{|c|c|c|c|c|c|}
\hline \multirow{2}{*}{$\begin{array}{l}\text { Clave del paisaje según } \\
\text { MORALES IGLESIAS } \\
\text { et al., (2017) }\end{array}$} & \multirow{2}{*}{$\begin{array}{l}\text { Tipo de } \\
\text { relieve }\end{array}$} & \multirow{2}{*}{$\begin{array}{l}\text { Rango de la } \\
\text { pendiente }\end{array}$} & \multirow{2}{*}{$\begin{array}{c}\text { Tipos de } \\
\text { vegetación o } \\
\text { usos de suelo }\end{array}$} & \multicolumn{2}{|c|}{ Área } \\
\hline & & & & $\mathrm{km}^{2}$ & $\%$ \\
\hline CXII.1 & PL(3) & Plana a & PC, AT. & 357.75 & 63.85 \\
\hline CXVIII.2 & $\mathrm{V}(3)$ & $\begin{array}{c}\text { ligeramente } \\
\text { inclinada }(<1 \mathrm{a} \\
\left.5^{\circ}\right)\end{array}$ & AT, AR, PC, PI. & 202.57 & 36.15 \\
\hline
\end{tabular}

Nota: PL: Planicie. V: Valle. 3: Complejo de colinas residuales, vegas y cauces. Fuente: Elaboración propia. 
FIGURA 3

Grados de transformación de la cobertura vegetal de los paisajes del estado de Chiapas, México

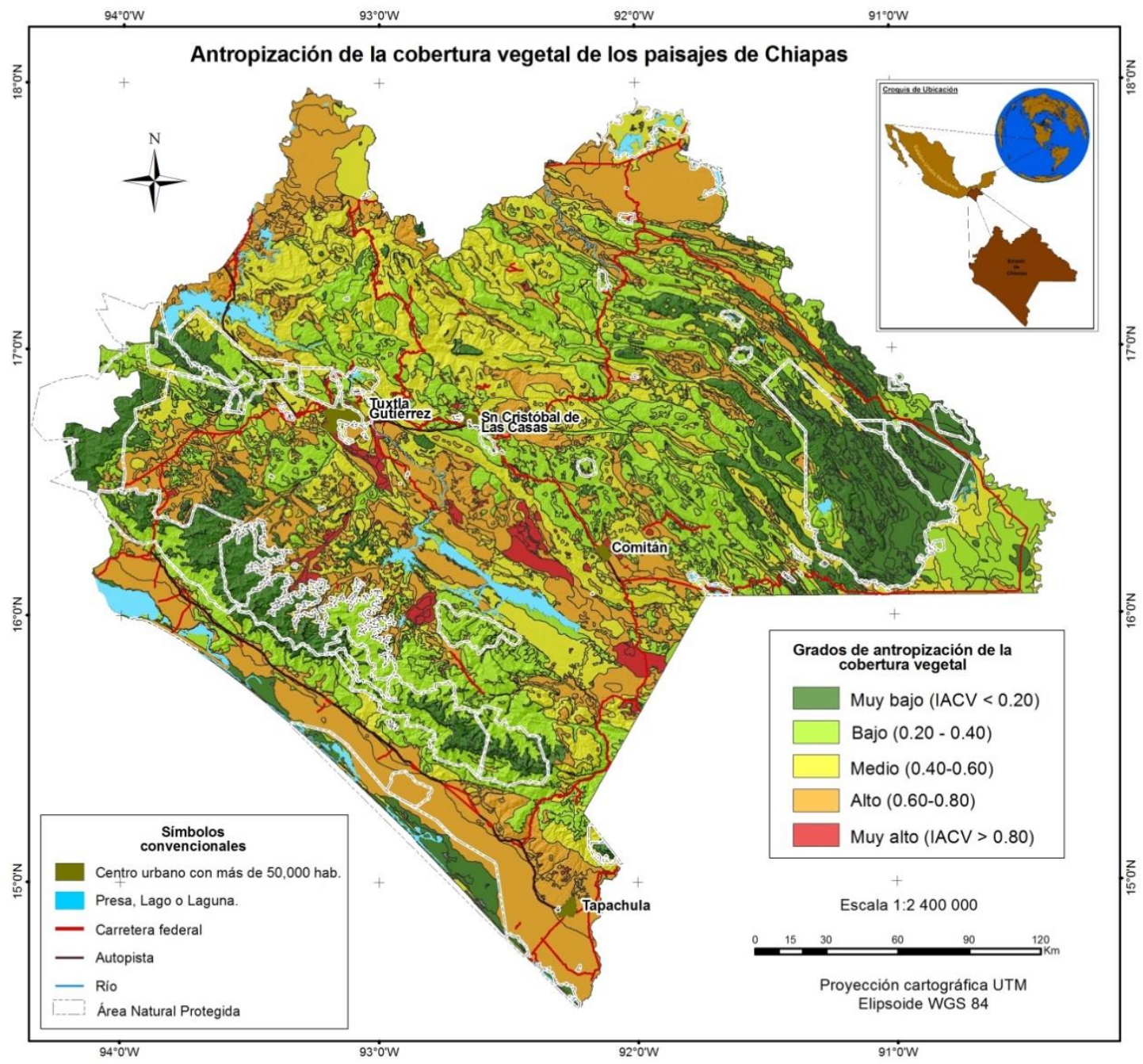

\subsection{LA ANTROPIZACIÓN DE LA COBERTURA VEGETAL EN LOS PAISAJES DE LAS ÁREAS NATURALES PROTEGIDAS (ANP's)}

De acuerdo con los resultados, la cobertura vegetal de la mayoría de los paisajes que integran las ANP's presentan bajos niveles de transformación; en este sentido, las categorías baja y muy baja del IACV ocupan $84 \%$ de la superficie; mientras que la categoría media abarca $11 \%$; finalmente, la categoría alta apenas representa 5\% (Figura 4 y Tabla 8). Los paisajes de categoría baja y muy baja establecen un predominio territorial en las siguientes ANP's: Montes Azules, La Sepultura, El Triunfo, La Frailesca, Selva El Ocote, y La Encrucijada. El componente morfológico de estos paisajes está constituido por montañas y planicies, las primeras reconocen un origen tectónico-intrusivo y tectónico-carsificado en climas semicálido y cálido húmedo a subhúmedo; mientras que las segundas son de tipo tectónico-acumulativo en clima cálido 
húmedo. Por su parte, los paisajes de planicie se desarrollan bajo un ambiente tectónicoacumulativo, en un clima cálido húmedo. Ambos escenarios morfogenéticos, permiten la presencia de bosque mesofilo de montaña, selva alta perennifolia y mediana subperennifolia.

Respecto a la categoría media, esta se establece principalmente en las siguientes ANP's: Sistema Lagunar Catazaja, Frailescana, Montes Azules; Selva El Ocote. Los paisajes más representativos están constituidos morfológicamente por montañas y planicies; en el primer caso, consideran un origen tectónico-intrusivo y tectónico-acumulativo, en climas cálido húmedo y cálido subhúmedo; mientras que los segundos poseen una génesis palustre, en clima cálido húmedo. Estas condiciones morfogenéticas favorecen la presencia de pastizales y agricultura.

En cuanto a la proporción territorial que representan las categorías del IACV con respecto a los paisajes ubicados dentro y fuera de las ANP's, destacan los niveles bajo y muy bajo; ya que una tercera parte de los paisajes que ostentan estas categorías se localizan dentro de estas.

TABLA 8

Superficie ocupada por las categorías del IACV en las ANP's

\begin{tabular}{|c|c|c|}
\hline Categoría & Superficie en $\mathrm{km}^{2}$ & Porcentaje \\
\hline Muy baja & 7,419 & 54 \\
\hline Baja & 4,069 & 30 \\
\hline Media & 1,537 & 11 \\
\hline Alta & 747 & 5 \\
\hline
\end{tabular}

Fuente: Elaboración propia

\subsection{ALGUNAS CONSIDERACIONES SOBRE LA ANTROPIZACIÓN DE LA COBERTURA VEGETAL DE LOS PAISAJES}

El análisis del presente estudio atiende una visión estatal a escala 1: 250 000; por ende, los resultados deben ser interpretados de manera cuidadosa. Bajo esta consideración, los niveles de antropización que posee la cobertura vegetal de los paisajes físico-geográficos obedecen a causas socio-económicas y no de índole natural. Por lo anterior, los paisajes fueron evaluados de manera independiente; es decir, sin tomar en cuenta el arreglo tipológico. Para cumplir con el objetivo fue indispensable el uso de herramientas SIG.

A pesar de lo anterior, los procesos de degradación geoecológica expresados a través de las categorías del IACV, muestran una relativa correspondencia espacial con la composición geomorfológica de los paisajes. Esta relación es más evidente en los Parajes Complejos constituidos por piedemonte, planicie y valle, debido a que manifiestan elevadas categorías; en cambio, la mayoría de los paisajes montañosos poseen bajos a muy bajos niveles de transformación.

Es posible considerar que el uso del territorio guarda cierto orden lógico, ya que los paisajes de baja energía de relieve, escasa pendiente, alta disponibilidad de agua, fácil acceso, así como favorables condiciones edáficas, incentivan usos de suelo agrícola, pecuario y urbano; por tanto, estos geosistemas representan los territorios de mayor asimilación socio-económica de la entidad.

Por su parte, los paisajes de categoría baja y muy baja se distinguen por poseer alta energía de relieve, elevada pendiente y menor accesibilidad; estas condiciones favorecen la presencia y conservación de las comunidades vegetales; por ende, es probable que alberguen altos niveles de bio y geodiversidad; a pesar de esto, la situación es preocupante, debido a que la vegetación secundaria ocupa una extensa superficie dentro de estos. Ante este escenario, los instrumentos de planificación territorial deben considerar una serie de políticas que permitan la conectividad de las comunidades bióticas; para ello, se deben proteger los corredores que conforman diversos paisajes en ladera y valle intermontano.

Por otra parte, los paisajes de categoría media tienen un papel relevante desde una perspectiva ambiental, ya que representan el punto de quiebre entre los paisajes de mayor y menor transformación geoecológica; por tanto, representan territorios sujetos a procesos de mayor 
transformación socio-económica, en donde, la sustitución de la vegetación natural por uso de suelo agrícola, pecuario, y urbano, significa el avance de la frontera agrícola; y con ello, la presencia de procesos asociados a la degradación geoecológica. Los paisajes de categoría media representan en muchas ocasiones un serio conflicto ambiental, ya que existe una discordancia $o$ incompatibilidad entre la composición natural de los paisajes y el uso de suelo; tal es el caso de los paisajes de laderas y barrancos ubicados en las cabeceras de las cuencas, en donde las condiciones geólogo-geomorfológicas generan una mayor susceptibilidad a procesos de remoción en masa. Ante esta situación, es posible considerar a los paisajes de categoría media como un escenario potencial de riesgo.

Ante esta situación, es prioritario construir nuevos esquemas sociales y económicos que establezcan una relación más armoniosa con la naturaleza; en este sentido, las prácticas agroecológicas, el eco y geoturismo, deben ser considerados como una alternativa de desarrollo para la entidad.

Respecto a los geosistemas de categoría muy alta, se recomienda mejorar el entorno ecológico a través de un desarrollo urbano ordenado, en donde se promueva el establecimiento de parques urbanos y evite el crecimiento sobre paisajes lacustres, valles fluviales y cabeceras de cuencas.

En cuanto a los paisajes de categoría baja y muy baja que forman las ANP's, destacan por ser parte fundamental de lo que se podría definir como sistema territorial de mayor patrimonio natural de Chiapas; sin embargo, la superficie que ocupan estos no es representativa, ya que la mayor parte de estos paisajes se encuentran fuera de las ANP's.

\section{CONCLUSIONES}

El IACV permitió conocer la composición de los cinco niveles de antropización de la cobertura vegetal de los paisajes de Chiapas. Bajo estas categorías se obtuvo una diferenciación espacial objetiva de Chiapas, que puede ser interpretada desde una perspectiva ambiental o económica; ya que identifica paisajes con un alto patrimonio natural, así como aquellos que representan un mayor desarrollo económico. Para cumplir con este objetivo, la herramienta SIG tuvo un papel preponderante.

Con base en lo anterior, se reconoce la relación geográfica que mantienen los niveles de antropización de la cobertura vegetal de los paisajes con el componente geológo-geomorfológico; en este sentido, los geosistemas con mayor grado de naturalidad consideran una composición montañosas de origen tectónico-intrusivo, tectónico-carsificado y tectónico-acumulativo, en climas semicálido y cálido húmedo a subhúmedo. Estos paisajes ocupan $45 \%$ de la superficie estatal, y se ubican principalmente en las regiones: Sierra Lacandona, Sierras del Sur de Chiapas, en partes de Montañas de Chiapas y Guatemala, y Volcanes de Centroamérica.

En cuanto a los niveles de degradación que manifiesta la cobertura vegetal de estos paisajes, es posible señalar que el difícil acceso y la baja densidad de población, son factores que han contribuido a la conservación de esta. Ante este panorama, los instrumentos de planificación ambiental deben considerar políticas que fomenten la conservación, preservación y protección de este patrimonio natural.

Por otra parte, la composición geomorfológica de los paisajes con mayor desarrollo económico está dada por planicies, piedemontes y valles, bajo un origen fluvial y fluviotorrencial, en climas semicálido y cálido húmedo a subhúmedo. Estos paisajes abarcan $29 \%$ del territorio chiapaneco, y se disponen principalmente por la Planicie Costera de Pacifico, Planicie Meridional del Golfo, y estribaciones septentrionales de las Montañas del Sur de Chiapas. En contraste al anterior grupo de paisajes, los presentes manifiestan una fácil accesibilidad, así como la presencia de importantes centros de población e infraestructura; esta situación genera una fuerte transformación de la cobertura vegetal. Ante este escenario, los planes de Ordenamiento Territorial y Ecológico deben establecer políticas ambientales que propicien un desarrollo sustentable.

Respecto a los paisajes de categoría media, el componente geomorfológico está integrado por un relieve montañoso de origen tectónico-intrusivo, tectónico-carsificado y tectónicoacumulativo, en climas semicálido húmedo a subhúmedo, cálido húmedo y cálido subhúmedo. Estos paisajes cubren 26\% del área estatal, y se distribuyen principalmente en la región Montañas 
del Norte de Chiapas. La deforestación, y la incompatibilidad entre la composición natural de estos paisajes (laderas con fuerte pendiente y suelos someros) con un uso de suelo agropecuario, generan el escenario ambiental más crítico de la entidad. Bajo este escenario de tendencia, es probable que 55\% de la superficie estatal presente en el corto plazo altos niveles de degradación, esto implicaría una mayor pérdida de bio y geodiversidad, una disminución de servicios ambientales, así como serios problemas asociados a los riesgos por fenómenos geólogogeomorfológicos. Ante esta situación, los instrumentos de planificación territorial deben considerar políticas ambientales dirigidas a la restauración.

Finalmente, las ANP's tienen un papel preponderante en la conservación del patrimonio natural de Chiapas; sin embargo, ante la incipiente superficie ocupada, cabe la posibilidad de considerar una transformación territorial de estas, así como la formulación de nuevas áreas; lo que conlleva a la generación de nuevos modelos sociales para la protección de la naturaleza.

\section{AGRADECIMIENTOS}

El primer autor agradece el apoyo del Instituto de Investigación en Gestión de Riesgos y Cambio Climático de la Universidad de Ciencias y Artes de Chiapas, y del Centro de Investigaciones en Geografía Ambiental de la Universidad Nacional Autónoma de México para el desarrollo de esta investigación. A Neftalí Aguilar Juárez y Aldo Saúl Gómez Parada por el apoyo en las etapas finales del proceso.

\section{BIBLIOGRAFÍA}

AGUiRRE, M. A. LÓPEZ IBARRA, L. I., BOLAÑOS TROCHEZ, F. V., GONZÁleZ GUEVARA, D. F. y O. BUITRAGO-BERMÚDEZ. (2017): Percepción del paisaje, agua y ecosistemas en la cuenca del río Dagua, Valle del Cauca, Colombia. Perspectiva Geográfica, 22(1), 109-126. doi: 10.19053/01233769.5402

BOLLO, M., HERNÁNDEZ, J.R., PRIEGO, A., ZARAGOZA, R., ORTÍZ, A., ESPINOZA, A., RUÍZ, R. (2015): Una propuesta de Regionalización físico-geográfica de México. Universidad Nacional Autónoma de México: Centro de Investigaciones en Geografía Ambiental. Morelia, Michoacán. 59 pp

BOLLO MANENT. M. (2018): La Geografía del Paisaje y la Geoecología: Teoría y enfoques. En: Checa-Artasu, M., Sunyer-Martín, P. Coord. El Paisaje: reflexiones y métodos de análisis. Ed. Ediciones del Lirio-Editorial UAM. México. ISBN: 978-607-28-1169-0

BOLLO MANENT, M. y VELASCO MÉNDEZ, W.E. (2018): El Estado del Medio Ambiente en Michoacán de Ocampo. México. Cuadernos Geográficos, 57(3), 118-139.

COSEJO NACIONAL DE POBLACIÓN (CONAPO). (1991): Sistema de ciudades y distribución espacial de la población en México. Tomo I. México D.F. 142 pp.

CRUZ PAZ, G., CASTILlO, M.M., ESPINOZA TENORIO, A., BRAVO PEÑA, L.C., VALENCIA BARRERA, E., M. MESA JURADO. (2018): Áreas prioritarias de conservación en la cuenca Usumacinta. La aplicación de un enfoque multicriterio. Investigaciones Geográficas. Instituto de Geografía. UNAM. (97), 1-19.

CUEVAS, M.L., GARRIDO, A., PÉREZ, J.L. D.I. GONZÁLEZ. (2010): Procesos de cambio de uso de suelo y degradación de la vegetación natural. En: Cotler, H. Coord. Las cuencas hidrográficas de México. Diagnóstico y priorización. Semarnat, INE y Fundación Gonzalo Río Arronte I.A.P. México, D.F. p: 96-103.

ESRI. (2012): ArcGIS Desktop. ESRI-GIS Mapping Software. ESRI Inc. CA, USA. http://www.esri.com

FLORES-DOMÍNGUEZ, A., RUÍZ-CAREAGA, J. A., A.G. PRIEGO-SANTANDER. (2017): Evaluación de la degradación de suelos en el estado de Puebla, México. En: Balochi, F. y Chreties, Ch. Edit. Procesos de erosión. Sedimentación en cauces y cuencas. UNESCO. Programa Hidrológico Internacional. América Latina y el Caribe. Volumen 3. Documento técnico No. 38. Paris. p: 80-96.

FROLOVA, M. (2006): Desde el concepto de paisaje a la teoría de geosistema en la Geografía rusa: ¿hacia una aproximación geográfica global del medio ambiente?. Ería, 70, pp: 225-235. 
HASSE, G. (1986): Theoretical and methodological foundations of landscape ecology. In: Landscape Ecology. Abstract of Lecture. International Training Course. Institute of Geography and Geoecology, GDR Academy of Sciencie, Leipzig, pp: 4 - 7.

INEGI. 2013: Conjunto de datos vectoriales de uso de suelo y vegetación escala 1:250 000, serie $\mathrm{V}$ (en línea). INSTITUTO NACIONAL DE ESTADÍSTICA Y GEOGRAFÍA. Aguascalientes, actualización: 15 de mayo de 2013, información cartográfica y bases de datos, https://www.inegi.org.mx/temas/usosuelo/default.html\#Descargas, acceso libre, [consulta: 28 de diciembre de 2019]

INEGI. 2018: Marco geoestadístico. INSTITUTO NACIONAL DE ESTADÍSTICA Y GEOGRAFÍA. https://www.inegi.org.mx/temas/mg/[consulta: 28 de diciembre de 2019].

ISACHENKO, A. G. (1973): Principles of landscape science and physical geographic regionalization. Trasl. R. J. Zatorski. Edit. J. S. Massey, Melbourne, 311 p.

MATEO-RODRÍGUEZ, J. (1984): Apuntes de Geografía de los Paisajes. Universidad de la Habana. Ministerio de Educación Superior. Ciudad de Habana. 470 p.

MATEO-RODRÍGUEZ, J. (2008): Geografía de los Paisajes. Primera parte. Paisajes Naturales. Editorial Universitaria. Ciudad de La Habana. ISBN 978-959-16-0730-0. 188 p.

MATEO-RODRÍGUEZ, J., ORTIZ-PÉREZ, M.A. (2001): La degradación de los paisajes como concepción teórico-metodológica. Serie Varia, Nueva Época, No. 1. Instituto de Geografía. UNAM. México, D. F. 40 p.

MORALES-IGLESIAS, H., PRIEGO-SANTANDER, A. G., M. BOLLO-MANENT. (2017): "Los paisajes físico-geográficos del estado de Chiapas, México a escala 1: 250 000". Terra Digitalis, international e-journal of map. 1 (1): 1-7.

MATHEWS, J. (2011): Evaluación de la modificación edafo-biógena de los paisajes en Michoacán, México. Investigación y Amazonía; 1(2), 78-84.

PRIEGO-SANTANDER, A., COTLER, H., FREGOSO, A., LUNA, N., C., ENRÍQUEZ. (2004): La dinámica ambiental de la cuenca Lerma-Chapala. Gaceta Ecológica nueva época. No.71. 23-38.

RODRÍGUEZ, M.L., LÓPEZ, J. (2008): Determinación de indicadores ambientales a escala detallada para la evaluación biofísica y la planeación del territorio: el caso de Milpa Alta, Distrito Federal. En: López, J., Rodríguez, M. Coords. Desarrollo de indicadores ambientales y de sustentabilidad en México. Instituto de Geografía, UNAM. Geografía para el siglo XXI. Serie Libros de Investigación. México, D.F. 165-196.

ROSETE-VERGÉS, F. A., PÉREZ-DAMIÁN, J.L., VILLALOBOS-DELGADO, M., NAVARRO-SALAS, E.N., SALINAS-CHÁVEZ, E., REMOND-NOA, R. (2014): El avance de la deforestación en México 1976-2007. Madera y Bosques, 20 (1): 21-35.

SEMENOV, Y.M. and V.A. SNYTKO. (2013): The 50th anniversary of the appearance of V.B. Sochava's first article on the geosystem. Geography and Natural Resources 34 (3): 197-200.

SEMENOV, Y. M. (2014): Landscape Geographical Support of the Ecological Policy of Nature Management in Regions of Siberia. Geography and Natural Resources 35 (3): 208-112.

SHAW, D.J.B. \& J. OLFIELD. (2007): Landscape science: a Russian geographical tradition. Annals of the Association of America Geographers, 97 (1): 111-126.

SHISHENKO, P.G. (1988): Estabilidad de los paisajes a las cargas económicas. Geografía Física Aplicada. Editorial de la Escuela Superior. Kiev, 195 p. 